\title{
Hyper-Spectral Imager in visible and near-infrared band for lunar compositional mapping
}

\author{
A S Kiran Kumar* and A Roy Chowdhury \\ Space Applications Centre, ISRO, Ahmedabad 380 015, India. \\ *e-mail: kiran@sac.isro.gov.in
}

India's first lunar mission, Chandrayaan-1, will have a Hyper-Spectral Imager in the visible and near-infrared spectral bands along with other instruments. The instrument will enable mineralogical mapping of the Moon's crust in a large number of spectral channels. The planned Hyper-Spectral Imager will be the first instrument to map the lunar surface with the capability of resolving the spectral region, 0.4 to $0.92 \mu \mathrm{m}$, in 64 continuous bands with a resolution of better than $15 \mathrm{~nm}$ and a spatial resolution of $80 \mathrm{~m}$. Spectral separation will be done using a wedge filter and the image will be mapped onto an area detector. The detector output will be processed in the front-end processor to generate the 64-band data with 12-bit quantization. This paper gives a description of the Hyper-Spectral Imager instrument.

\section{Introduction}

The Hyper-Spectral Imager (HySI) instrument operating in visible and near-infrared band is planned for India's Chandrayaan-1 satellite for mapping the lunar surface along with other imaging payloads. The uniqueness of this instrument will be its capability of mapping in 64 contiguous bands in the spectral range of 0.4 to $0.92 \mu \mathrm{m}$ with a spectral resolution of better than $15 \mathrm{~nm}$ and spatial resolution of $80 \mathrm{~m}$. The high spatial and spectral resolution data of HySI along with NIR payloads onboard Chandrayaan-1 namely, SMART-1 Infra-red Imager (SIR-2) (Nathues et al 2000) and Moon Mineralogy Mapper (M3) will significantly improve upon the available mineral compositional information. Study of deep regions like South Pole-Aitken basin, which represents lower crust or upper mantle material, will also help in understanding the composition and mineralogy of the interior of the Moon and its formation and evolution. The HySI is conceived as a highly compact instrument keeping with the mission requirement of low weight, size and power. This paper gives a description of the planned HySI instrument.

\section{Operating principle}

The instrument will map the lunar surface in push broom-mode from a polar orbit of $100 \mathrm{~km}$ altitude. HySI will collect the Sun's reflected light from the Moon's surface through a tele-centric refractive optics and focus on to an area detector. One frame will correspond to $40 \mathrm{~km}$ along-track by $20 \mathrm{~km}$ across-track area on ground. The $20 \mathrm{~km}$ across-track corresponds to the swath coverage. Rectangular frame size is to improve the spectral resolution. The pixel footprint is a uniform square. Wedge filter is planned for spectral dispersion. The advantage of wedge filter over prism/grating is the implementation simplicity and the compactness. The wedge filter will be placed in close proximity of the detector for spectral separation of the input light corresponding to a frame. The filter will be placed so as to have the spectral change in the along-track direction. The rows of the detector will thus be receiving irradiance corresponding to different areas in the along-track direction and also the spatial information of the rows will be in different spectral bands. For example, data of the $N$ th row of the detector covering swath $S_{N}$ will be in

Keywords. Hyper-Spectral Imager; wedge filter; spectral bands; mineral mapping.

J. Earth Syst. Sci. 114, No. 6, December 2005, pp. 721-724

(C) Printed in India. 


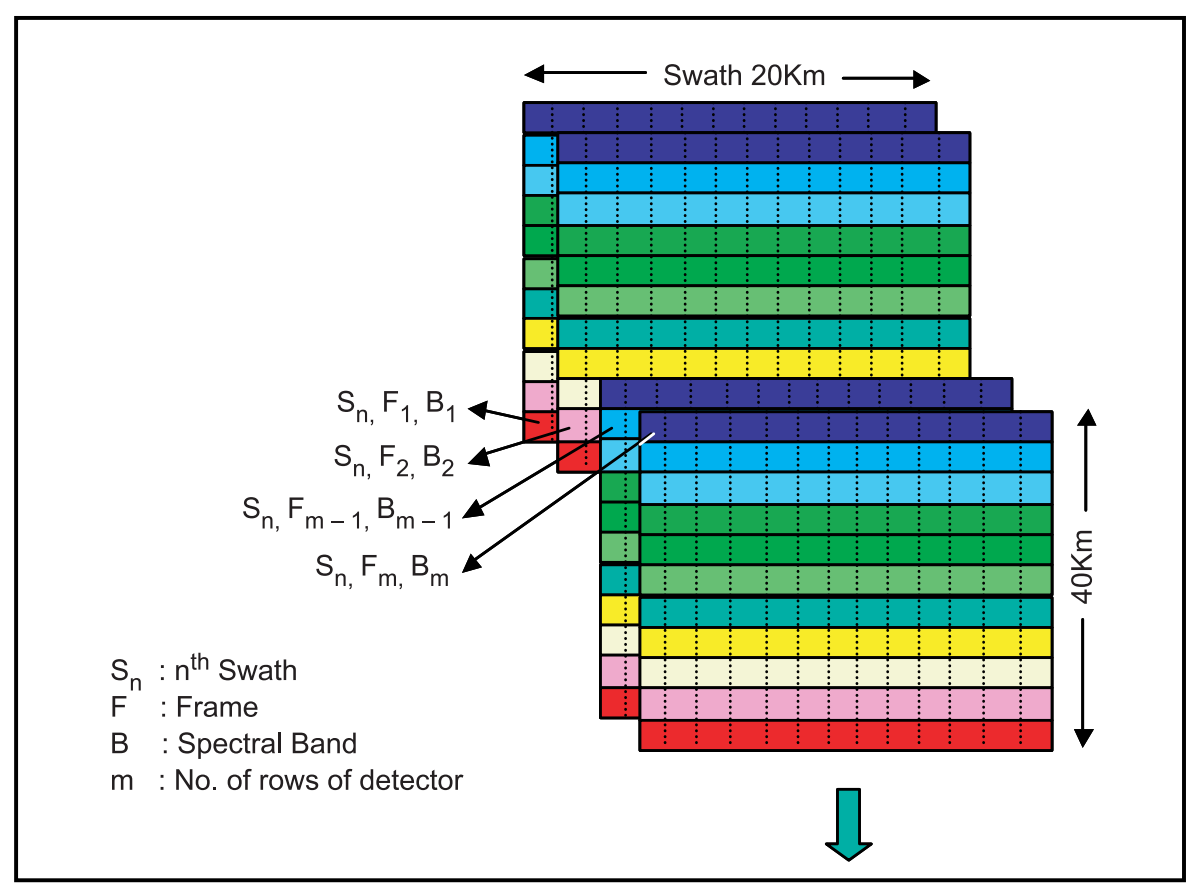

Figure 1. Mapping scheme adopted for Hyper-Spectral Imager.

Table 1. Specifications of Hyper-Spectral Imager.

\begin{tabular}{rll}
\hline 1. & Ground resolution $(\mathrm{m})$ & $\begin{array}{c}80 \mathrm{~m} \text { from } 100 \mathrm{~km} \\
\text { altitude }\end{array}$ \\
2. & Swath $(\mathrm{km})$ & $\sim 20$ \\
3. & Spectral range $(\mu \mathrm{m})$ & $0.4-0.92$ \\
4. & No. of spectral bands & 64 contiguous \\
5. & Spectral resolution $(\mathrm{nm})$ & $<15$ \\
6. & No. of gains & 2 \\
7. & Quantization (bits) & 12 \\
8. & SNR $\left( \pm 60^{\circ}\right.$ lat. for mature & $>100$ \\
9. & mare soil) & 16 \\
10. & Estimated power $(\mathrm{W})$ & 3 \\
\hline
\end{tabular}

the spectral band $B_{N}$ and the data of the subsequent row $N+1$ th will correspond to swath $S_{N+1}$ and spectral band $B_{N+1}$. The pixels of a row in the across track direction will receive irradiance in the same spectral band separated spatially to cover the swath. The mapping is shown pictorially in figure 1.

The spectrally over sampled data available from the detector will be processed onboard to generate the 64 bands for reducing the transmittable data volume. Adding pixel data along the roll axis from the over sampled frames corresponding to the same object area will generate the 64 bands. The radiometric resolution of the instrument will be 12 bits to cover the large signal variation of the Moon's surface with a single gain. This will facilitate imaging the high and low illumination scenes simultaneously. In addition, provision will be there to operate the instrument at a higher gain by ground command. The features of the HySI payload are given in the table 1 .

\section{Hardware description}

The HySI instrument will comprise of focusing optics, wedge filter for spectral separation, detector, camera electronics and the mechanical housing. The instrument will be made in two units, with the first unit comprising of optics, wedge filter and the detector. The second unit will be the camera electronics connected to the first unit through an interconnecting harness.

The collecting optics is designed as refractive, tele-centric with five elements lens assembly to cover a circular field of view of $\pm 13^{\circ}$ in the spectral range of 0.4 to $0.92 \mu \mathrm{m}$. It will be an $\mathrm{f} / 4$ system with an effective focal length of $62.5 \mathrm{~mm}$. The area detector will be placed in the focal plane. The wedge filter for spectral separation will be placed in close proximity of the detector. The preliminary schematic of this unit is shown in figure 2 .

The detector planned for the instrument is an Active Pixel Sensor (APS) area array with a 10bit digitized output for reduction of external hardware. The configuration of the camera electronics is based on the detector and system requirements. The system performance will be limited by photon noise and no MTF degradation or band-toband mis-registration will be introduced by the processing hardware. The electronics will provide 


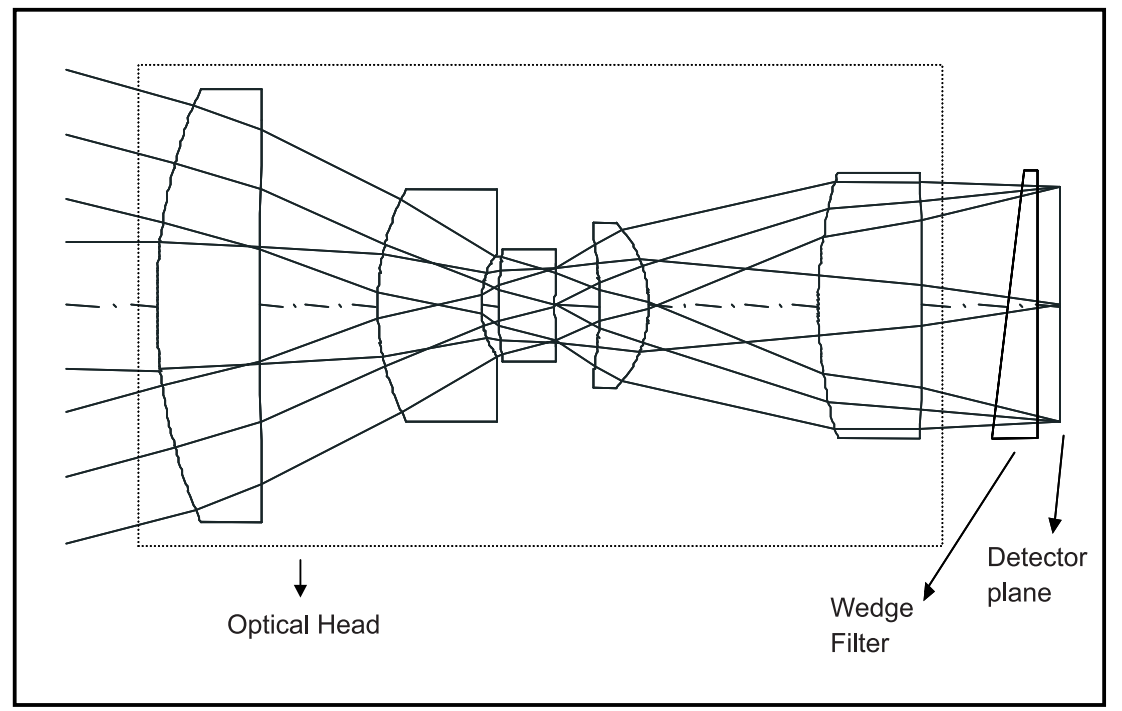

Figure 2. Schematic of Hyper-Spectral Imager first unit.

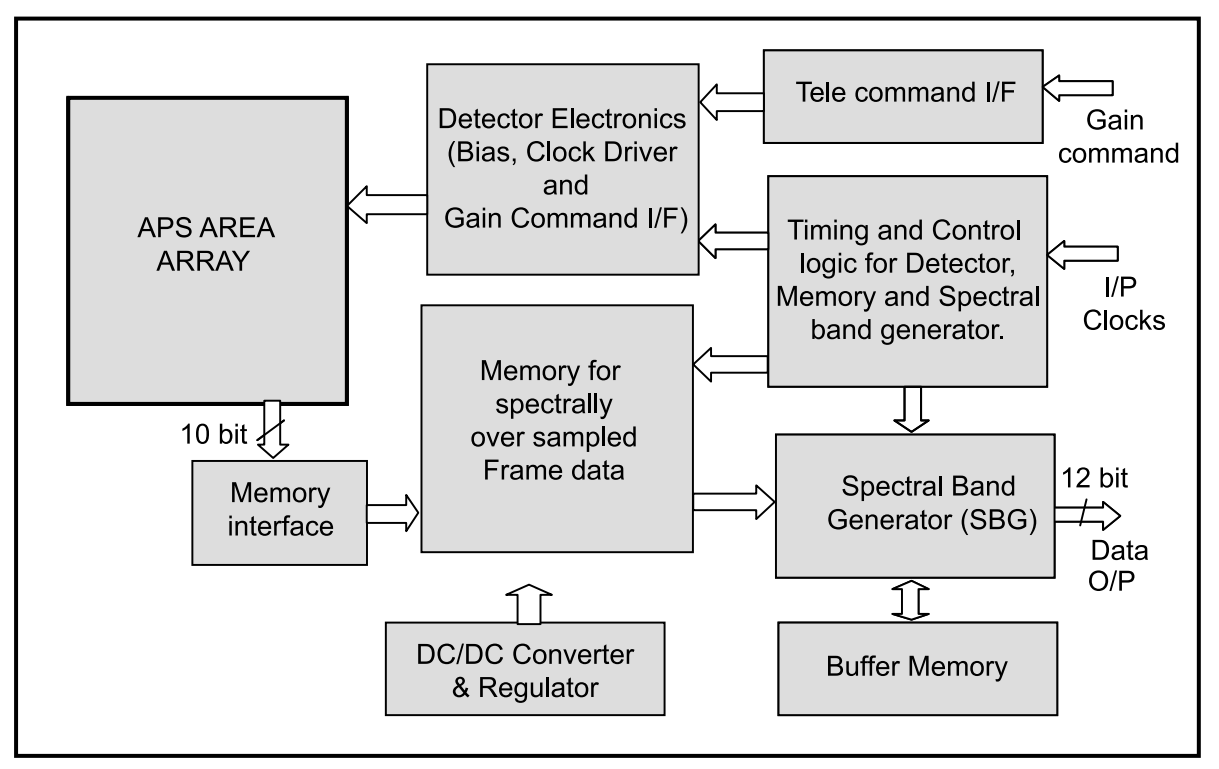

Figure 3. Schematic of Hyper-Spectral Imager Camera electronics.

the input stimulus to the detector for its operation and process the digitized output to generate the 64-band data. The spectrally over sampled frames will be stored in memory. The 64 bands will be generated by adding the pixel data from the stored spectrally over sampled frames, corresponding to the same object area. The output will be 12-bit quantized data. The preliminary block schematic of the electronics is shown in figure 3 .

The instrument is packaged in two units to minimize the thermal effects on the optics and detector. The estimated weight of the instrument will be about $3 \mathrm{~kg}$, keeping with the mission requirement of weight and size minimization and meeting the functional and performance requirements. The preliminary conceptual instrument view is shown in figure 4.

\section{Conclusions}

With the Hyper-Spectral Imager, complete mapping of the Moon will be possible in 64 bands in the visible and near-infrared region with a spectral resolution of better than $15 \mathrm{~nm}$ for scientific studies. On ground the instrument will be spatially, radiometrically and spectrally calibrated to units of incident radiance. In-flight verification may be done in the overlapping band regions with other optical payloads in infrared camera SIR-2 and Moon 


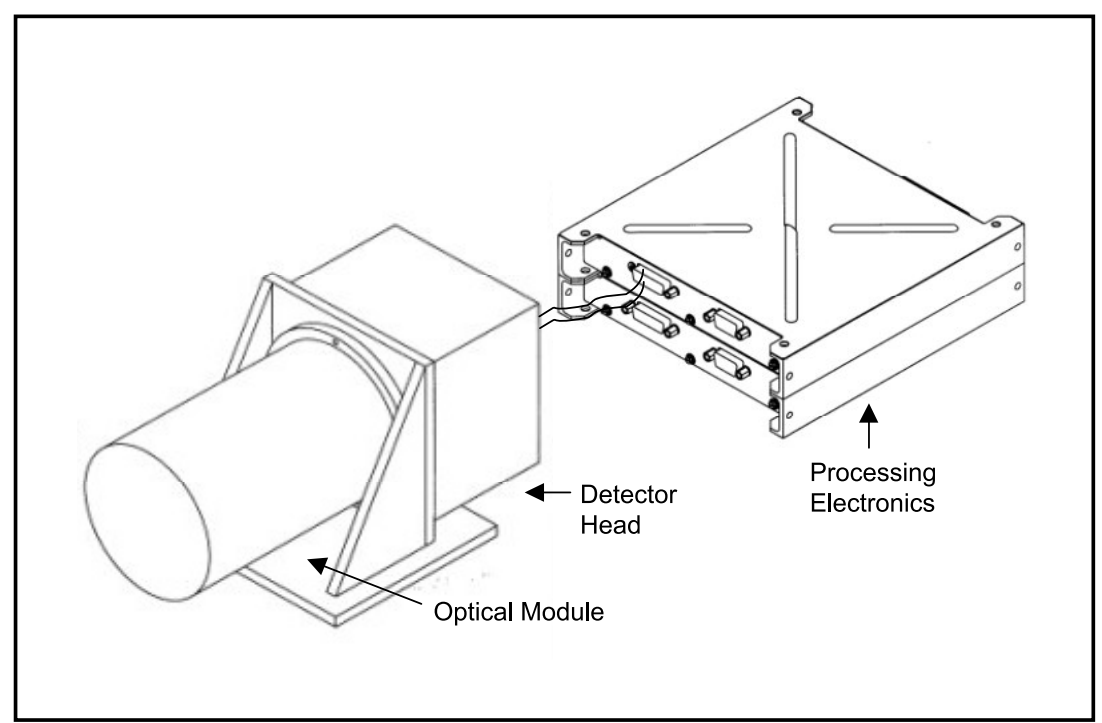

Figure 4. Schematic of Hyper-Spectral Imager instrument.

Mineral Mapping (M3). The mineralogical information of the complete lunar surface available from Chandrayaan- 1 is expected to improve the available data on the Moon.

\section{Acknowledgements}

Authors acknowledge with thanks the contributions from D R M Samudraiah, Saji Kuriakose, Chirag Dewan, Kurien Mathew, S S Sarkar,
S R Joshi and Sanjeev Mehta. We also gratefully acknowledge the encouragement and guidance received from Dr. K N Shankara, Director, and Dr. Ram Rattan, Deputy Director Space Applications Centre.

\section{References}

Nathues A, Mall U and Keller U 2000 Near-Infrared Spectrometry with SIR on Smart-1; Proc. Fourth Int. Conf. on the Exploration and Utilization of the Moon 101-103. 